\title{
Colistin: our last line of defence
}

\section{Jennifer Coetzee ${ }^{\mathrm{a}^{*}}$}

aAMPATH Pathologists, Cape Town, South Africa

*Corresponding author, email: coetzeej@ampath.co.za

The polymyxins are a group of antibiotics which were first isolated from a spore-bearing soil bacillus, Bacillus polymyxa, in 1947. Colistin, also known as polymyxin E, was introduced into clinical practice over 50 years ago. Although it has remained available for use since then, it was largely shelved in the 1970s with the discovery of antibiotics that were equally or more effective but considered less toxic.'

In the words of Prof. Helen Giamarellou, colistin is an antibiotic that "should have stayed on the shelf". However, with the emergence of multidrug-resistant (MDR) and extensive drugresistant (XDR) Pseudomonas aeruginosa and Acinetobacter baumannii combined with the decline in the discovery of new antibiotics, it is often the only active antibiotic available to the clinician. Recently, the emergence and unprecedented spread of the carbapemases, including NDM-1, OXA-48 and VIM, has further lead to a worldwide increase in the use of colistin as salvage therapy.

Multidrug-resistant organisms (MROs) have a high capacity to develop resistance to antibiotics. As colistin was licensed for use in the 1960s, it was never subjected to the drug development processes that are standard for regulatory compliance today. The dosing data was based on microbiological assays that were used to measure concentrations of colistin in fluids. These dosing strategies from the 1960s are, therefore, unlikely to be effective in the era of MDR and XDR organisms with ever increasing minimum inhibitory concentrations (MICs). ${ }^{2}$ The clinical failure rates of colistin for the treatment of these MROs, therefore, have been expectedly high, with rates as high as $47.2 \%$ reported. ${ }^{3}$ In a study by Falagas, ${ }^{4}$ higher daily colistin doses were associated with increased survival, highlighting the need to optimise the dosing of colistin in critically ill patients. The publication of the South African Society for Clinical Pharmacists' guideline for colistin dosing may contribute to improving the outcomes of patients in South Africa.

It was inevitable that the increased use of colistin would result in the emergence of colistin resistance. In South Africa, the first colistin-resistant Klebsiella pneumoniae was described in 2012, following a failed attempt at selective digestive decontamination. The resistance was due to a single amino acid change in protein PmrB, which arose simultaneously in strains from three different continents. ${ }^{6}$ In a case-control study, previous colistin therapy was associated with an increased risk of bloodstream infection with colistin-resistant $K$. pneumoniae, along with previous colonisation with KPC-producing K. pneumoniae and a Charlson score of $\geq 3$. $^{7}$

Even more concerning is the recent identification of the plasmidmediated $\mathrm{mcr}^{-1}$ gene encoding for resistance to colistin. This transmissible gene was identified in Escherichia coli isolates from animal sources (farm pigs, retail pork and chicken) from China, and is expected to emulate other global resistance mechanisms such as the carbapenemases. A few weeks after the initial report was published, the Lancet Infectious Diseases published a spate of reports from countries all over the world reporting the presence of $m c r-1$ in colistin resistant isolates. ${ }^{8-11}$ In South Africa, the presence of $m c r-1$ in clinical $E$. coli isolates from patients has now been confirmed and will be presented in an upcoming publication (Ampath, personal data). In contrast to the colistinresistant $K$. pneumoniae isolates, the $E$. coli isolates are not multidrug resistant, and were isolated from both hospitalised patients as well as patients from the community. While these isolates still have numerous treatment options, the concern is that the plasmid will spread to other isolates where this might no longer be the case, resulting in pan-drug resistant organisms.

In conclusion, the use of colistin must be restricted in hospitals in South Africa. A hospital's consumption of colistin is a surrogate marker of the efficacy of its antimicrobial stewardship and infection prevention efforts, which should be considered standard care. The alarming spread of the $\mathrm{mcr}^{-1}$ gene in the food supply, and on to human pathogens, has already prompted calls for a global ban on the use of colistin in agriculture. Failing to do so may expedite the loss of efficacy of this 'last line of defence'.

\section{References}

1. Li J, Nation Roger L, Turnidge JD, et al. Colistin: the re-emerging antibiotic for multidrug-resistant Gram-negative bacterial infections. Lancet Infect Dis. 2006;6:589-601.

2. Roberts JA, Lipman J. Editorial commentary: closing the loop--a colistin clinical study to confirm dosing recommendations from PK/ PD modeling. Clin Infect Dis. 2012;54(12):1727-1729.

3. Tzouvelekis LS, Markogiannakis $A$, Psichogiou $M$, et al. Carbapenemases in Klebsiella pneumoniae and other enterobacteriaceae: an evolving crisis of global dimensions. Clin Microbiol Rev. 2012;25(4):682-707.

4. Falagas ME, Rafailidis PI, loannidou $\mathrm{E}$, et al. Colistin therapy for microbiologically documented multidrug-resistant gram-negative bacterial infections: a retrospective cohort study of 258 patients. Int J Antimicrob Agents. 2010;35:194-199.

5. Brink AJ, Coetzee J, Corcoran C, et al. Emergence of OXA-48 and OXA181 carbapenemases among enterobacteriaceae in South Africa and evidence of in vivo selection of colistin resistance as a consequence of selective decontamination of the gastrointestinal tract. J Clin Microbiol. 2013;51(1):369-72.

6. Jayol A, Poirel L, Brink A, et al. Resistance to colistin associated with a single amino acid change in protein PmrB among Klebsiella pneumoniae isolates of worldwide origin. Antimicrob Agents Chemother. 2014;58(8):4762-4766.

7. Giacobbe DR, Del Bono V, Trecharichi EM, et al. Risk factors for bloodstream infections due to colistin-resistant KPC-producing Klebsiella pneumoniae: results from a multicenter case-control-case 